\title{
Estudo de mercado de iogurte da cidade de Belo Horizonte/MG
}

\author{
Milene Moreira Ribeiro, Valéria Paula Rodrigues Minim², Luis Antônio Minim ${ }^{3}$, Aline Cristina Arruda ${ }^{4}$ Elaine \\ Berger Ceresino ${ }^{5,}$ Helena Cristina Ferrer Carneiro ${ }^{5,}$ Paula de Aguiar Cipriano
}

\section{RESUMO}

O estudo do perfil dos consumidores e dos fatores envolvidos no processo de compra do iogurte é uma ferramenta importante na identificação dos diferentes segmentos desse mercado e de suas potencialidades. Assim, neste trabalho entrevistaram-se 387 consumidores de iogurte, residentes em Belo Horizonte - MG, que responderam os questionários estruturados. Os resultados foram analisados de forma descritiva, e para verificar a correlação entre características sociodemográficas com o perfil de consumo aplicou-se a análise de correlação de Pearson. Os resultados indicaram que o iogurte sabor morango apresenta maior aceitação pelos consumidores, que a categoria light/diet possui grande parte do seu público consumidor (54\%). Segundo a pesquisa, 39,9\% dos respondentes que não consomem iogurte light/diet disseram não consumi-lo por não gostar desse produto. Isto revela a importância de pesquisas para desenvolvimento de iogurte light/diet visando à melhoria da qualidade sensorial. O fato de não ver necessidade de consumir o produto foi apontado como um dos principais motivos por não consumir iogurte da categoria light/diet. A qualidade, a marca e o preço apresentaram grande importância na conquista de mercado, sendo os três principais atributos observados no momento da compra de iogurte na cidade estudada. Analisando a correlação entre consumo e fator sociodemográfico, observou-se que a renda e a idade influenciam tanto na frequência de consumo de iogurte quanto no consumo do produto na versão light/diet.

Palavras-chave: Iogurte, consumidores, pesquisa de mercado.

\section{ABSTRACT}

\section{Yogurt market research in the city of Belo Horizonte, Brazil}

The study of consumers' profile and the factors involved in the moment of purchasing yogurt is an important tool for identifying different market segments and their potentialities. Therefore, the aim of this study was to interview 387 yogurt consumers residing in the city of Belo Horizonte - MG, Brazil, using structured questionnaires. The results were analyzed in a descriptive way. Pearson's correlation was applied in order to verify the correlation between sociodemographic characteristics and the consumers' profile. The results indicated that strawberry flavored yogurt shows the greatest acceptance among consumers and the category of light/diet represents a great part of the consumers' preferences (54\%). According to the survey, 39,9\% of the respondents that do not consume light/diet yogurts stated that they do not appreciate these products. These results shows the importance of carrying out research to develop light/diet yogurt with improved sensorial quality. One of the main reasons pointed out for consumers not to eat light/ diet yogurt is that they do not see the need for consuming it. In the city the research took place, quality, brand and price

Recebido para publicação em maio de 2009 e aprovado em janeiro de 2010

${ }^{1}$ Engenheira de alimentos, Mestre. Universidade Federal de Viçosa, Departamento de Tecnologia de Alimentos, Av. PH Rolfs, s/n, Campus universitário, 36570-000, Viçosa, Minas Geais, Brasil. Milene.ribeiro@gmail.com

${ }^{2}$ Engenheira de alimentos, Doutora. Universidade Federal de Viçosa, Departamento de Tecnologia de Alimentos, Av. PH Rolfs, s/n, Campus universitário, 36570-000, Viçosa, Minas Geais, Brasil.vprm@ufv.br

${ }^{3}$ Engenheiro de alimentos, Doutor. Universidade Federal de Viçosa, Departamento de Tecnologia de Alimentos, Av. PH Rolfs, s/n, Campus universitário, 36570-000, Viçosa, Minas Geais, Brasil. Iminim@ufv.br

${ }^{4}$ Economista doméstica, Doutora. Universidade Federal de Viçosa, Departamento de Tecnologia de Alimentos, Av. PH Rolfs, s/n, Campus universitário, 36570-000, Viçosa, Minas Geais, Brasil. acarrudaufv@yahoo.com.br

${ }^{5}$ Engenheiro de alimentos. Universidade Federal de Viçosa, Departamento de Tecnologia de Alimentos, Av. PH Rolfs, s/n, Campus universitário, 36570-000, Viçosa, Minas Geais, Brasil. 
had the greatest importance in the act of purchasing, since these were the three main attributes reported to have been observed in the moment of the purchase.The analysis of the correlation between consumption and socio-demographic factors showed that income and age influence both the frequency of yogurt consumption and the consumption of the light/diet version of the product.

Key words: Yogurt, consumers, market research.

\section{INTRODUÇÃO}

O iogurte é um dos poucos alimentos conhecidos e consumidos a mais de 4.500 anos. A Bulgária foi um dos primeiros países a consumi-lo, e o divulgou para o restante do mundo. Nos anos 1950 teve sua popularidade aumentada ao considerá-lo um alimento bom para a saúde e para o corpo. Desde então, esse produto foi ganhando espaço no dia a dia, passando a fazer parte dos hábitos alimentares de muitas pessoas (Moraes, 2004). Apesar da grande variedade de sabores e marcas disponíveis no mercado brasileiro, o consumo per capita de iogurte no Brasil é de apenas $3 \mathrm{~kg}$ por ano, valor ainda pequeno quando comparado a países como França, Uruguai e Argentina, onde o consumo per capita do produto é de 7 a $19 \mathrm{~kg}$ ao ano (Bolini \& Moraes, 2004).

O mercado total de iogurte no Brasil movimentou 2 bilhões de reais em 2005. A categoria de lights, que representa em média $10 \%$ desse montante, cresceu $11 \%$ em 2006 (Gazeta, 2006).

A demanda por produtos light e diet tem crescido muito e tende a aumentar ainda mais. Nos últimos 10 anos, o mercado de light e diet registrou aumento de $870 \%$, segundo a Associação Brasileira da Indústria de Alimentos Dietéticos e para Fins Especiais (ABIAD). Só em 2004, o crescimento foi de cerca de $25 \%$. Para 2005, a ABIAD previu um crescimento ainda maior, próximo de $30 \%$ (Domenich, 2005). As vendas de produtos denominados light e diet corresponderam, em 2005, a cerca de 7 bilhões de dólares, apresentando crescimento de $800 \%$ nos últimos 10 anos. Hoje, 35\% das famílias brasileiras consomem algum tipo desses produtos (Abiad, 2007). Esse crescimento se deve a um aumento na preocupação na relação entre dieta e saúde, estimulando o consumo de alimentos saudáveis, nutritivos e funcionais ou, ainda, de baixo valor calórico, que tenham nutrientes com potencial protetor de saúde.

Outro fator que favoreceu o aumento no consumo de produtos light/diet foi a nova imagem dos consumidores frente às palavras light/diet, que eram associadas apenas às pessoas que tinham problemas de saúde ou queriam perder peso, que passaram a abranger também aos consumidores que prezam por um corpo saudável e em boa for- ma. Segundo Abiad (2007), essa mudança no público vem acontecendo com o segmento de bebidas lácteas de baixa caloria, principalmente os iogurtes.

Para satisfazer às necessidades dos consumidores é preciso conhecer as pessoas, seus desejos e suas necessidades. Para compreender como e porquê as pessoas compram é importante o estudo do comportamento do consumidor (Cobra, 2006). As técnicas de pesquisa de mercado podem auxiliar no desenvolvimento de produtos como um mecanismo de captação das necessidades dos consumidores, monitorando seus hábitos e suas atitudes, além de avaliar os protótipos dos produtos (Polignano, 2001). Assim, a pesquisa de mercado é grande aliada na conquista de mercado de produtos light/diet.

Este trabalho teve como objetivos obter o perfil dos consumidores de iogurte residentes na cidade de Belo Horizonte/MG, os sabores consumidos, os principais atributos observados na compra do produto, a frequência de consumo de iogurte, o consumo de iogurte light/diet e as razões pela opção de consumir ou não esses produtos.

\section{MATERIAL E MÉTODOS}

A amostragem foi definida de acordo com o público consumidor de iogurte. Para determinar o tamanho da amostra utilizou-se a fórmula para amostras finitas (Mattar,1997):

$$
n=\frac{N Z^{2} p \cdot q}{e^{2}(N-1)+Z^{2} p \cdot q}
$$

em que:

$\mathrm{n}=$ tamanho da amostra;

p = proporção de ocorrência da variável em estudo na população;

q = considerando a característica representada por "p", este é a proporção de não-ocorrência ( $p=1-q)$;

$\mathrm{N}$ = número de elementos da população;

Z = valor da variável z para o nível de confiabilidade adotado; e

e = precisão da amostra ou erro máximo admitido

Estabeleceu-se o nível de significância de e $=5 \%$. Dessa forma, o valor de Z adotado é de 1,96. Quando as 
proporções de ocorrência da variável estudada na população não são conhecidas, pode-se adotar $\mathrm{p}=\mathrm{q}=0,50$. $\mathrm{O}$ tamanho da população foi obtido nos dados do IBGE de 2006, sendo a população de Belo Horizonte igual a $\mathrm{N}=$ 2399920. Assim, o tamanho da amostra foi de no mínimo 385 consumidores em Belo Horizonte.

No período de 21 a 30 de março de 2007, 387 consumidores foram entrevistados em dois supermercados de grande circulação em Belo Horizonte. Os respondentes foram abordados aleatoriamente durante a compra do produto estudado. Dessa forma, foi possível atingir a população de diversos perfis sociais que consomem iogurte caracterizando os consumidores de iogurte da cidade estudada.

A pesquisa de mercado foi realizada por meio da aplicação de questionário aos consumidores composto de questões fechadas. Esse foi elaborado com linguagem simples, procurando obter o máximo de informações possíveis sobre o perfil dos consumidores e suas preferências. O questionário foi composto por duas etapas, sendo a primeira formada por questões socioeconômicas e a segunda, pelo perfil de consumo do iogurte (Tabela 1). A fim de evitar resultados tendenciosos, optou-se por utilizar cartões circulares contendo todas as respostas, para que o respondente apontasse a sua opção. Teve-se o cuidado de variar a posição do cartão ao apresentá-lo.

Após a aplicação dos questionários, eles foram conferidos, numerados e tabulados, visando à posterior análise e interpretação dos dados. Os fatores sociais, idade e renda, foram correlacionados por meio da Análise de Correlação de Pearson com a frequência de consumo de iogurte e com o consumo de iogurte light/diet, A correlação de Pearson fornece a intensidade e a direção da interação entre duas variáveis (Malhotra et al, 2006). Para a tabulação e análise dos dados foi utilizado o programa SPSS (Statistical Package for the Social Sciences), versão 15.0.

\section{RESULTADOS E DISCUSSÃO}

Os 387 entrevistados tinham idade variando de 14 a 87 anos, sendo $69,8 \%$ do sexo feminino e $30,2 \%$ do sexo masculino. A idade média dos entrevistados foi de 38 anos, e o desvio-padrão, de 14,6 anos. Os resultados revelaram predominância de consumidores entre 20 e 40 anos, o que corresponde a 55,6\% dos entrevistados, caracterizando o mercado de iogurte pela prevalência de consumidores adultos.

Dos entrevistados, 50,9\% declararam ser casados e $40,8 \%$ solteiros. A escolaridade média foi o superior completo, 38,5\%, e 63,3\% dos entrevistados afirmaram ter cursado ou estarem cursando o ensino superior. Com relação à renda, 24,3\% dos respondentes declararam receber até $\mathrm{R} \$ 1.350,00$ por mês e 18,1\% responderam ter renda mensal superior a $\mathrm{R} \$ 7.851,00$. Entretanto, a maioria dos entrevistados, 57,6\%, apresentou renda mensal bem distribuída nos valores intermediários, de $\mathrm{R} \$ 1.351,00$ a R\$7.850,00. O perfil sociodemográfico dos consumidores de iogurte residentes na cidade de Belo Horizonte está apresentado na Figura 2.

Com relação ao consumo, 93,7\% dos entrevistados disseram consumir iogurte pelo menos uma vez por semana (Figura 3a) e 54,0\% utilizram o produto na versão light/ $\operatorname{diet}$ (Figura 3b).

Os principais motivos apontados por esse grupo para optar pelo iogurte na versão light/diet foram para manter a forma (55,2\%), por gostar do produto $(18,1 \%)$ e por indicação médica (17,1\%) (Figura 4a). Hara (2003) obteve resultado semelhante no estudo de mercado de produtos light/diet no qual "manter a forma” foi a principal razão apontada pelos consumidores para a utilização desses produtos.

Os entrevistados que não consomem iogurte light/ diet alegaram ser outro motivo não apresentado no questionário (50\%) e por não gostar do produto (39,9\%) os principais fatores que limitam o consumo (Figura 4b). A maioria dos entrevistados que apresentaram outro motivo para não utilizar produtos light/diet alegou não ver necessidade de consumir esses produtos.

Os resultados sugerem que os iogurtes light e diet são direcionados ao público de maior poder aquisitivo. A aceitação sensorial do produto demonstrou afetar o consumo de produtos light/diet, confirmando a importância de desenvolver produtos dessa categoria com características sensoriais semelhantes às do produto tradicional.

Tabela 1. Resumo das questões apresentadas aos consumidores de iogurte.

\begin{tabular}{ll}
\hline & Conteúdo das questões \\
\hline I etapa* & \multicolumn{1}{c}{ II etapa* } \\
\hline Sexo & Sabor de iogurte mais consumido \\
Data de nascimento & Atributos observados na compra do iogurte \\
Estado civil & Frequência de consumo de iogurte \\
Grau de instrução & Consome iogurte light ou diet \\
Renda familiar mensal & Por que consome light ou diet \\
& Por que não consome light ou diet \\
\hline
\end{tabular}

\footnotetext{
* Questões fechadas com respostas de acordo com cada pergunta.
} 
Idade
Sexo
Estado Civil

Escolaridade

Renda $\geq 60$ anos

50-59

40-49

30-39

20-29

$\leq 19$ anos

Feminino

Masculino

Divorciado

Viúvo

União estável

Solteiro

Outros

Pós-graduação completa Pós-graduação incompleta Superior completo

Superior incompleto $2^{\circ}$ grau completo

$2^{\circ}$ grau incompleto

$1^{\circ}$ grau completo

$1^{\circ}$ grau incompleto

Acima de $\mathrm{R} \$ 7.851,00$

R\$ 7.351,00 a R\$ 7.850,00

RS $6.851,00$ a RS $7.350,00$

$R \$ 6.351,00$ a R\$ $6.850,00$

$\mathrm{R} \$ 5.851,00$ a $\mathrm{R} \$ 6.350,00$

R\$ $5.351,00$ a R\$ $5.850,00$

RS $4.851,00$ a R\$ $5.350,00$

RS $4.351,00$ a R\$ $4.850,00$

R\$ $3.851,00$ a R\$ $4.350,00$

RS $3.351,00$ a R\$ $3.850,00$

R\$ $2.851,00$ a R\$ $3.350,00$

$R \$ 2.351,00$ a R\$ $2.850,00$

R\$ $1.851,00$ a R\$ $2.350,00$

R\$ $1.351,00$ a R\$ $1.850,00$

$\mathrm{R} \$ 851,00$ a R\$ $1.350,00$

$R \$ 350,00$ a $R \$ 850,00$

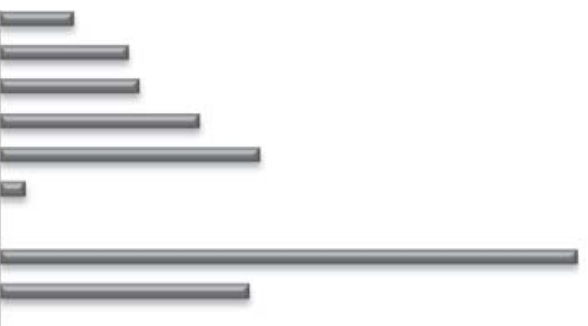

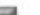
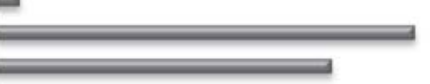

.
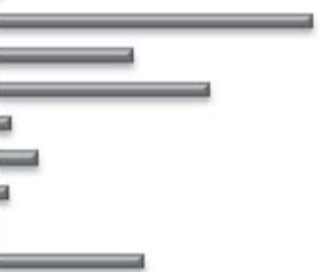

$=$

可

Figura 2. Perfil sociodemográfico dos consumidores de iogurte da cidade de Belo Horizonte/MG.

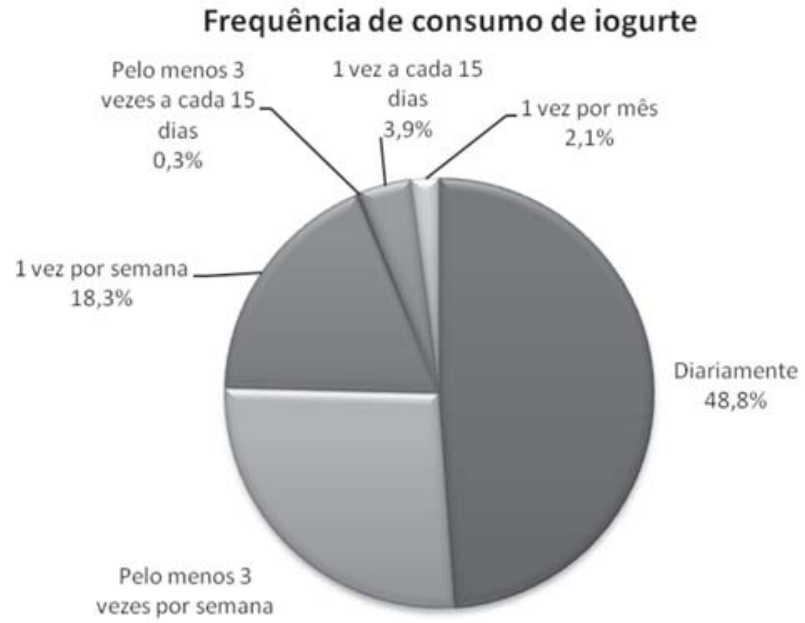

$$
26,6 \%
$$

(a)

\section{Consumo de iogurte light ou diet}

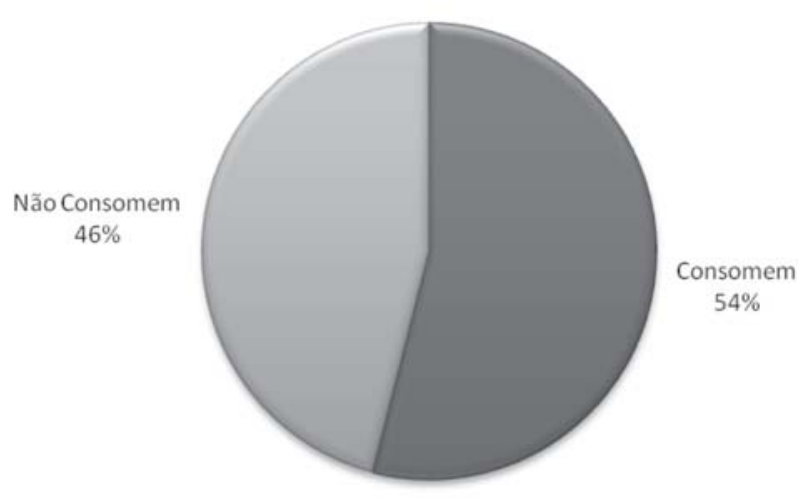

(b)

Figura 3. Freqüência de consumo de iogurte (a) e parcela do mercado que consome o produto na versão light/diet (b) na cidade de Belo Horizonte

Rev. Ceres, Viçosa, v. 57, n.2, p. 151-156, mar/abr, 2010 


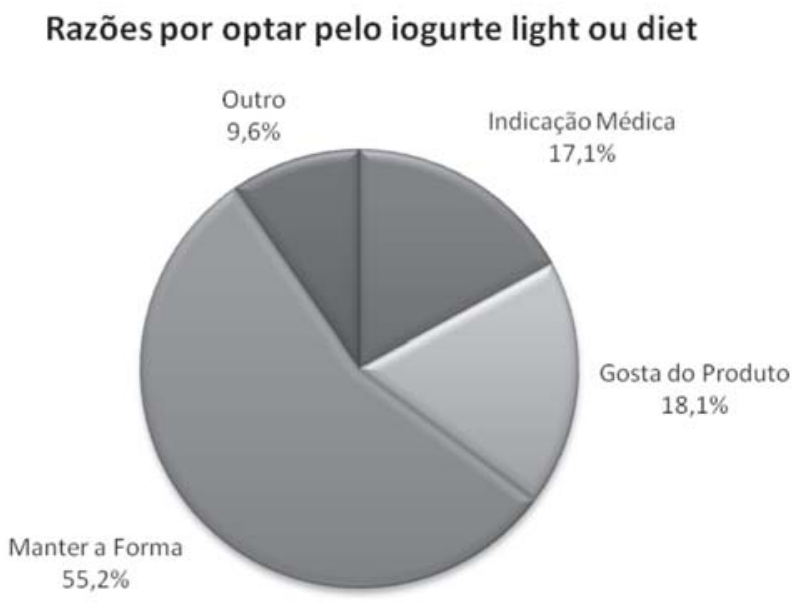

(a)

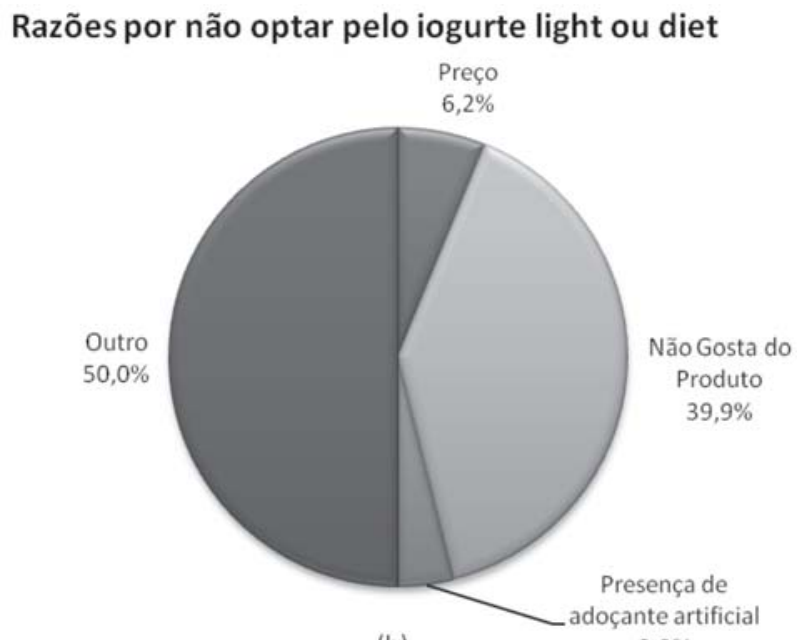

(b) $3,9 \%$

Figura 4. Motivos apontados pelos entrevistados em Belo Horizonte por consumir (a) ou não consumir (b) iogurtes light/diet

Quanto ao sabor, 58,1\% dos entrevistados indicaram o de morango como a primeira opção e 9,0\% deles declararam consumir a bebida somente desse sabor. Dos consumidores que não optaram pelo sabor morango como primeira opção, 15\% o escolheram como a segunda e 4,1\% como a terceira. De acordo com Reis (2007) atualmente existem diferentes sabores de iogurte disponíveis no mercado brasileiro, mas o de morango representa cerca de 70 $80 \%$ do volume de vendas do País. A Figura 5 apresenta os principais sabores escolhidos pelos consumidores de iogurte da cidade de Belo Horizonte.

Os entrevistados foram questionados quanto aos principais atributos observados na hora da compra do produto. Segundo Cobra (2006), o consumidor, diante de diversas informações, se depara com a necessidade de escolher a melhor alternativa em termos de conveniência e custo, sofrendo ainda a influência da marca. Neste estudo 72,8, 51,7 e 49,6\% dos entrevistados disseram verificar o preço, a qualidade e a marca, respectivamente, como os três primeiros atributos observados (Figura 6). Cobra (2006) afirma

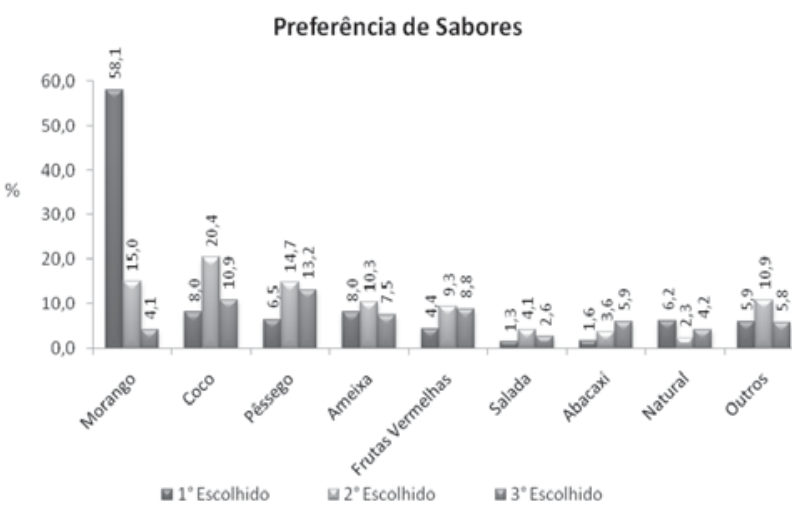

Figura 5. Principais sabores escolhidos pelos consumidores de iogurte da cidade de Belo Horizonte. ainda que quando o fator preço for importante, o produto de melhor custo e qualidade ganha a preferência.

Foi realizada a Análise de Correlação de Pearson a fim de investigar a correlação entre os fatores sociais, idade e renda, com os fatores de consumo, frequência de consumo de iogurte e consumo de iogurte light/diet (Tabela 2).

Pode-se observar que a renda correlaciona-se positivamente ( $\mathrm{p}<0,01)$ com a frequência de consumo de iogurte e com o consumo desse produto light/diet. O aumento na renda implica no aumento do consumo de iogurte em geral e de iogurte light/diet para a população estudada. Isso comprova que o preço elevado dos produtos light/diet é o principal fator para exclusão dessa categoria da lista de produtos consumidos pelo público de menor poder aquisitivo. Sugere-se ainda que o iogurte light/ diet é direcionado ao público de maior poder aquisitivo

O consumo de iogurte light/diet correlaciona-se positivamente $(\mathrm{p}<0,01)$ com a idade, indicando que o uso dessa categoria aumenta com a idade. Isso provavelmente se deve às necessidades de dieta de saúde que normalmente

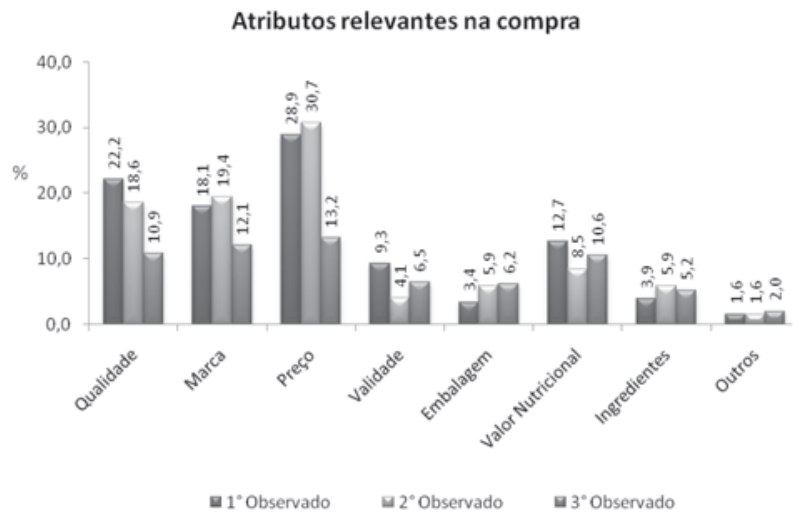

Figura 6. Atributos relevantes na compra de iogurte pelos consumidores da cidade de Belo Horizonte 
Tabela 2. Correlação entre fatores sociais e de consumo dos consumidores de iogurte da cidade Belo Horizonte

\begin{tabular}{lccc}
\hline Fatores Sociais & Fatores de consumo & $\begin{array}{c}\text { Frequência de consumo } \\
\text { de iogurtes }\end{array}$ & $\begin{array}{c}\text { Consumo de iogurte } \\
\text { light/diet }\end{array}$ \\
\hline \multirow{2}{*}{ Idade } & Correlação de Pearson & 0,201 & 0,142 \\
& Significância & $<0,001$ & 0,005 \\
\multirow{2}{*}{ Renda } & Correlação de Pearson & 0,197 & 0,189 \\
& Significância & $<0,001$ & $<0,001$ \\
\hline
\end{tabular}

surgem na terceira idade, sendo a indicação médica um dos principais motivos apresentados pelos consumidores para o consumo dessa categoria. Cobra (2006) afirma que a idade da pessoa exerce forte influência no seu tipo de consumo. Em cada faixa etária, o grupo de consumidores tem um estilo de vida e isso predispõe um tipo de consumo.

A idade e a frequência de consumo de iogurte correlacionam-se positivamente $(\mathrm{p} \leq 0,01)$. Nesse caso, quanto maior a idade maior a frequência de consumo de iogurte, o que confirma a predominância do público adulto no consumo.

A pesquisa realizada em Belo Horizonte demonstrou que o iogurte light/diet de sabor morango apresenta grande mercado potencial, uma vez que iogurtes de baixa caloria representam uma parcela significativa do mercado nessa cidade, e o sabor morango foi o preferido pelos consumidores entrevistados.

\section{CONCLUSÕES}

A pesquisa realizada demonstrou que o iogurte sabor morango apresenta maior aceitação pelos consumidores entrevistados. A categoria de iogurte light/diet tem grande mercado, abrangendo 54\% de seus consumidores. Apesar disso, nota-se a necessidade de investir em pesquisa a fim promover melhorias na qualidade sensorial do iogurte dessa categoria, pois 39,9\% dos entrevistados que não consome tais produtos disseram não consumi-los por não gostar.

A qualidade, a marca e o preço apresentaram grande importância na conquista de mercado, sendo os três principais atributos observados no momento da compra de iogurte na cidade estudada.

A pesquisa demonstrou que para alcançar o público consumidor da cidade de Belo Horizonte a publicidade deve ser voltada para pessoas adultas, destacando as vantagens do produto na manutenção da forma, beleza e longevidade. É necessário substituir a imagem dos produtos diet/light como alimento para pessoas doentes por outra que transmita importância na qualidade de vida, independentemente da indicação médica. A publicidade dos iogurtes da categoria diet/light deve ser direcionada ao público de maior poder aquisitivo.

\section{AGRADECIMENTOS}

Ao CNPq e à FAPEMIG, pelo apoio financeiro. Aos supermercados Extra e Supernosso, por ceder espaço para a realização das entrevistas.

\section{REFERÊNCIAS}

Abiad (2007) Associação Brasileira das Indústrias de Alimentos Dietéticos e para Fins Especiais. Disponível em:<http:// www.abiad.org.br >. Acessado em: 13 de agosto 2007.

Bolini HMA \& Moraes P (2004) Tese mostra que análise sensorial incrementaria produção de iogurte. Jornal da Unicamp, 253:11.

Cobra M (2006) Administração de Marketing no Brasil. 2 Ed. São Paulo, Cobra. p.454.

Domenich M (2005) Diet e light engordam faturamento. SEBRAE/ SC - Oportunidade de Negócios.. Disponível em: <http:// www.sebrae-sc.com.br>. Acessado em: 13 de agosto 2007.

Gazeta Mercantil (2006) Iogurte light quer conquistar consumidor que não faz dieta. Notícias: Laticínios.net. Disponível em:<http:/ /www.laticinio.net/noticias>. Acessado em: 14 de agosto 2007.

Hara CM (2003) O perfil do Consumidor de produtos light e diet no mercado de varejo supermercadista de Campinas. Revista Técnica FIPEP, 3:39-48.

IBGE (2006) Instituto Brasileiro de Geografia e Estatística. Disponível em: <http://www.ibge.gov.br/cidadesat/topwindow.htm $>$. Acessado em: 15 de março de 2006.

Malhotra NK, Rocha I, Laudisio MC, Altheman F \& Borges FM (2006) Introdução à Pesquisa de Marketing. São Paulo, ABDR. 428p.

Mattar FN (1997) Pesquisa de marketing : metodologia, planejamento, V1. 4a ed. São Paulo, São Paulo: Atlas. 336p.

Moraes PCBT (2004) Avaliação de iogurtes líquidos comerciais sabor morango: estudo de consumidor e perfil sensorial. Dissertação de Mestrado Universidade Estadual de Campinas, Campinas. $128 \mathrm{p}$.

Polignano LAC (2001) O papel da pesquisa de mercado durante o desenvolvimento de produtos. In: $3^{\circ}$ Congresso Brasileiro de Gestão de Desenvolvimento de Produtos, Florianópolis. Anais, UFSC, p.121-130. CD-ROM.

Reis RC (2007) Iogurte “light” sabor morango: equivalência de doçura, caracterização sensorial e impacto da embalagem na intenção de compra do consumidor. Tese de Doutorado. Universidade Federal de Viçosa, Viçosa. 143p. 